\title{
P07-10. Natural compounds in bodily fluids which bind DC-SIGN and prevent HIV-I capture and transfer to CD4 cells MJ Stax ${ }^{2}$, G Pollakis ${ }^{2}$, RR Sprenger ${ }^{1}$, M Melchers ${ }^{2}$, T van Montfort ${ }^{2}$, RW Sanders ${ }^{2}$, D Speijer ${ }^{1}$ and WA Paxton*2
}

\author{
Address: ${ }^{1}$ Department of Medical Biochemistry, Amsterdam, Netherlands and ${ }^{2}$ Laboratory of Experimental Virology, Academic Medical Center, \\ Amsterdam, Netherlands \\ * Corresponding author
}

from AIDS Vaccine 2009

Paris, France. 19-22 October 2009

Published: 22 October 2009

Retrovirology 2009, 6(Suppl 3):PI08 doi:I0.II86/1742-4690-6-S3-PI08

This abstract is available from: http://www.retrovirology.com/content/6/S3/PI08

(c) 2009 Stax et al; licensee BioMed Central Ltd.

\section{Background}

An effective HIV-1 vaccine will have to interfere with viral transmission across a mucosal surface and prevent the establishment of infection. The exact mechanism of the infectious process at the mucosa is still poorly understood as are the effects of bodily fluids on HIV-1 transmission.

\section{Methods}

We have studied the effects of human milk (HM) and seminal plasma (SP) on interfering with the capture of HIV-1 through binding to DC-SIGN on dendritic cells and prevent the subsequent transfer of HIV-1 to CD4 cells. HM and SP were tested in Raji-DC-SIGN and immature dendritic cell transfer assays to determine interference with HIV-1 capture and transfer. Fractionation and MALDI-TOF biochemical analysis was utilized to identify proteins with DC-SIGN binding activity.

\section{Results}

We identify that both HM and SP can potently block the capture and transfer of HIV-1 through DC-SIGN. Through our analysis we show that bile-salt stimulated lipase (BSSL) in human milk and mucin6 in seminal plasma have the capacity to inhibit HIV-1 transfer. BSSL is a highly variable protein with its variant genotypic forms showing different capacities to bind and inhibit. We also demonstrate that there is a large degree of variation between individuals for their HM or SP to prevent binding. Both these proteins have similarities in structure and in their specific glycosylation patterns which likely facilitate their binding efficiencies.

\section{Conclusion}

We identify that BSSL and mucin6 have the capacity to bind DC-SIGN and prevent HIV-1 capture at mucosal surfaces. By understanding their function and role in viral transmission we can learn more on the infection process but more importantly develop agents with either HIV-1 blocking capacity or which can target antigen at mucosal surfaces to dendritic cells and for use as effective adjuvants with mucosal vaccines. 